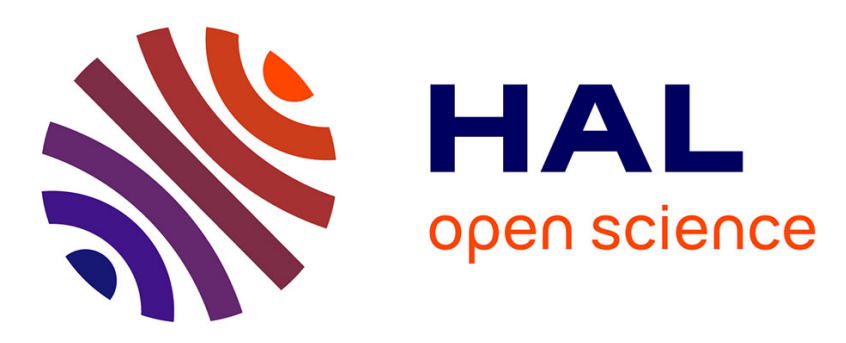

\title{
Long coherence times for Rydberg qubits on a superconducting atom chip
}

\author{
C. Hermann-Avigliano, R. Celistrino Teixeira, T. L. Nguyen, T. \\ Cantat-Moltrecht, Gilles Nogues, I. Dotsenko, S. Gleyzes, J. M. Raimond, S. \\ Haroche, M. Brune
}

\section{To cite this version:}

C. Hermann-Avigliano, R. Celistrino Teixeira, T. L. Nguyen, T. Cantat-Moltrecht, Gilles Nogues, et al.. Long coherence times for Rydberg qubits on a superconducting atom chip. Physical Review A : Atomic, molecular, and optical physics [1990-2015], 2014, 90 (4), pp.040502. 10.1103/PhysRevA.90.040502 . hal-01052819

\section{HAL Id: hal-01052819 https://hal.science/hal-01052819}

Submitted on 13 Feb 2015

HAL is a multi-disciplinary open access archive for the deposit and dissemination of scientific research documents, whether they are published or not. The documents may come from teaching and research institutions in France or abroad, or from public or private research centers.
L'archive ouverte pluridisciplinaire HAL, est destinée au dépôt et à la diffusion de documents scientifiques de niveau recherche, publiés ou non, émanant des établissements d'enseignement et de recherche français ou étrangers, des laboratoires publics ou privés. 


\title{
Long coherence times for Rydberg qubits on a superconducting atom chip
}

\author{
C. Hermann-Avigliano, ${ }^{1}$ R. Celistrino Teixeira,${ }^{1}$ T.L. Nguyen,${ }^{1}$ T. Cantat-Moltrecht,${ }^{1}$ \\ G. Nogues, ${ }^{2}$ I. Dotsenko, ${ }^{1}$ S. Gleyzes,${ }^{1}$ J.M. Raimond,${ }^{1}$ S. Haroche,${ }^{1}$ and M. Brune ${ }^{1}$ \\ ${ }^{1}$ Laboratoire Kastler Brossel, ENS, UPMC-Paris 6, CNRS, \\ Collège de France, 11 place Marcelin Berthelot, 75005, Paris, France. \\ ${ }^{2}$ Univ. Grenoble Alpes, Inst. NEEL, F-38042 Grenoble, France.
}

(Dated: February 13, 2015)

\begin{abstract}
Superconducting atom chips and Rydberg atoms are promising tools for quantum information processing operations based on the dipole blockade effect. Nevertheless, one has to face the severe problem of stray electric fields in the vicinity of the chip. We demonstrate a simple method circumventing this problem. Microwave spectroscopy reveals extremely long coherence lifetimes (in the millisecond range) for a qubit stored in a Rydberg level superposition close to the chip surface. This is an essential step for the development of quantum simulation with Rydberg atoms and of a hybrid quantum information architecture based on atomic ensembles and superconducting circuits.
\end{abstract}

PACS numbers: 03.67.-a,32.80.Ee,32.30.-r

Rydberg atoms [1] are the focus of a renewed interest. They are nearly ideal tools for the exploration of fundamental quantum behaviors [2] or for the implementation of quantum information processing [3] and quantum simulation $[4,5]$.

The dipole-dipole interaction [6] provides a strong long-range coupling between Rydberg atoms. It results in the dipole blockade phenomenon [7], which precludes the excitation of more than one Rydberg atom in a small sample. Preparation of samples with a precisely determined Rydberg atom number [8, 9] and ordered structures [10-12] have been proposed and demonstrated. The dipole blockade also leads to efficient quantum gates $[13,14]$ and to optical nonlinearities at the single photon level [15].

The transitions between Rydberg levels are very sensitive to millimeter-wave fields, with frequencies comparable to those used in circuit quantum electrodynamics [16]. One may envision a hybrid quantum processing architecture, unifying superconducting artificial atoms with an optical field interface based on dipole blockade [1719]. This leads us towards the use of cold atom clouds tightly trapped on superconducting atom chips. Moreover, superconducting chips offer a very long trap lifetime $[20,21]$, leading to an easy production of BoseEinstein condensates [22]. Rydberg atoms, which have been trapped in optical lattices [23], can also be trapped on-chip [24].

Rydberg atoms near a chip set a formidable experimental challenge, due to their sensitivity to static stray electric fields (Stark effect) [25, 26]. The patch effect due to adsorption of residual gas [27] or of alkali atoms onto the chip $[28,29]$ is particularly harmful. In particular, each cooling and trapping sequence releases a large number of alkali atoms, which stick onto the chip. They create a patch of dipoles, resulting in inhomogeneous electric fields, which destroy atomic coherences and evolve on an hourly basis. This jeopardizes the practical use of alkali
Rydberg atoms on a chip [30-33] unless this problem can be circumvented. The stray field control methods proposed so far either do not apply to alkali deposits [27] or require specific conditions, which might not be compatible with most experiments $[29,34]$.

We demonstrate in this paper the coherent manipulation of Rydberg atoms by microwave spectroscopy near a chip surface. We observe coherence times in the millisecond range. We get rid of the stray field problem by simply covering the front surface of the chip with a thin metallic Rubidium layer, making further uncontrolled atomic depositions harmless. These results open a promising avenue for the use of dipole blockade in quantum simulation and hybrid quantum information processing.

Figure 1(a) presents the experimental system (for details see [22]). The chip is held vertically in a ${ }^{4} \mathrm{He}$-cooled cryostat at $4.2 \mathrm{~K}$. Rubidium- 87 atoms are sent upwards towards the chip from a 2D-MOT source (provided by the SYRTE - Système de Référence Temps Espace - laboratory). They are caught, a few millimeters away from the chip, in a mirror-MOT, whose quadrupolar magnetic field is provided by centimeter-sized superconducting coils. The mirror-MOT uses two $780 \mathrm{~nm}$ laser beams [red arrows on figure 1(a)] reflected on the front gold surface of the chip and two others, counter-propagating in the $x$ direction (see axis orientations in Fig. 1).

The cold atom cloud is then transferred into an onchip mirror-MOT. Figure 1(b) presents a scheme of the chip. The conducting lines are etched by Reactive Ion Etching out of a $1.7 \mu \mathrm{m}$-thick Niobium film deposited on an oxidized silicium substrate. The $U$-shaped wire (width $300 \mu \mathrm{m}$ ), connecting the $J$ and $L$ pads, used together with a uniform field bias creates the quadrupole field for the on-chip MOT.

The atoms are then cooled down to $12 \mu \mathrm{K}$ by an optical molasses, and optically pumped in the $5 S, F=2, m_{F}=2$ state. They are transferred into a Ioffe-Pritchard magnetic trap, whose field is generated by a uniform bias and 

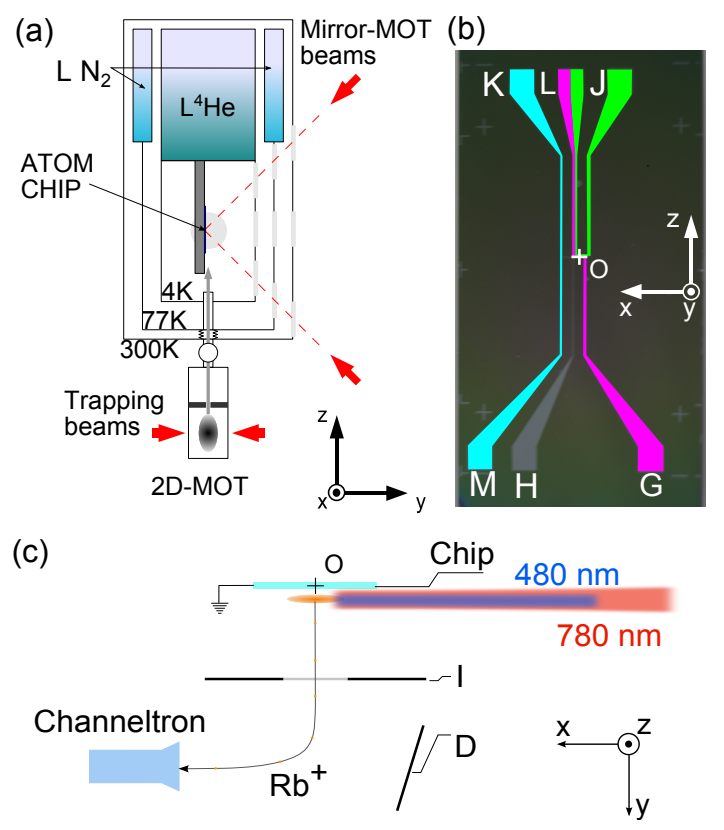

FIG. 1. (color online) . (a) Scheme of the experimental setup. (b) Scheme of the superconducting atom chip. The letters $G$ to $M$ label the current input pads on the chip. (c) Scheme of the field ionization detection system. The field-ionization electrode is $I$. The electrode $D$ deflects the ions towards the channeltron counter. Note the axes definition in the three panels. The origin $O$ is taken at the center of the horizontal segment of the $Z$-wire connecting pads $G$ and $L$. Note that the axis are shown offset with respect to this origin for clarity.

the $Z$-shaped wire (width: $70 \mu \mathrm{m}$ ), connecting pads $G$ and $L$. The high critical current (3.6 A) and the reduced length of the horizontal portion of this $Z$-wire make it possible to achieve tight trapping. The field in the bottom of the trap is aligned along the $x$ quantization axis.

An ancillary wire, connecting pads $K$ and $M$, feeds a radio-frequency field for evaporative cooling, optionally leading to Bose-Einstein condensation [22]. We operate here with moderately cooled thermal clouds to avoid density effects [35]. The $B_{x}$ magnetic field at the bottom of the trap is measured directly by RF spectroscopy.

The position of the atomic cloud in the $z$ and $y$ direction can be changed by adjusting the bias field. The cloud-to-chip distance, $y$, can be varied from $80 \mu \mathrm{m}$ to $700 \mu \mathrm{m}$. The evaporation is performed at $y=80 \mu \mathrm{m}$ and the cloud is then adiabatically moved and expanded towards its final position.

We then excite the two-photon $5 S \rightarrow 60 S$ transition with a $780 \mathrm{~nm}$ beam and a frequency-doubled diode (Toptica TA-SHG-110) at $480 \mathrm{~nm}$. The detuning with respect to the intermediate $5 P_{3 / 2}$ level is $540 \mathrm{MHz}$. The 'red' and 'blue' lasers, with waists of 150 and $22 \mu \mathrm{m}$ respectively, propagate along the $x$ axis [Fig. 1(c)], with $\sigma_{+}$ and $\sigma_{-}$polarizations. They excite the $60 S_{1 / 2}, m_{j}=1 / 2$ sublevel. Both lasers are frequency-locked to a transfer

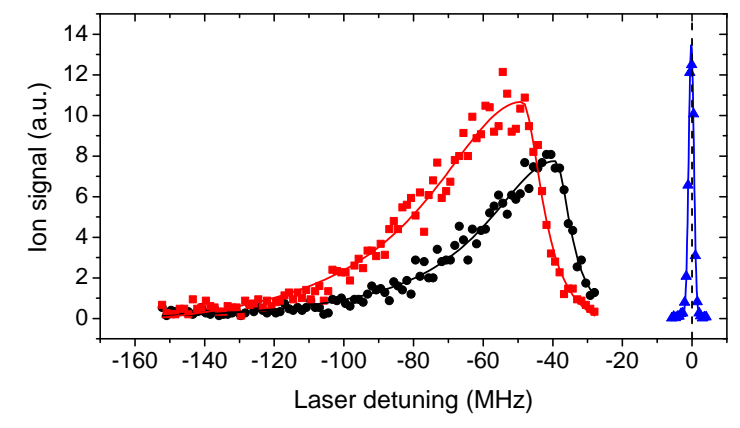

FIG. 2. (color online). Laser spectroscopy of the $5 S-60 S$ two-photon transition. The ion signal (arbitrary units) is shown as a function of the blue laser detuning w.r.t. the expected line position in zero field (dashed vertical line). The black and red/gray points are taken at a 40 minutes time interval in a MOT at $y=550 \mu \mathrm{m}$ from the chip with a gold front mirror. The respective line widths are 30 and $40 \mathrm{MHz}$. The asymmetric Lorentzian line shapes are guides to the eye. The blue triangles show the laser line after the Rubidium metallic layer deposit, in a magnetic trap at $y=670 \mu \mathrm{m}, z=0$. The solid line is a Gaussian fit, with a $1.7 \mathrm{MHz}$ FWHM.

Fabry Perot cavity, whose length is stabilized to a Rubidium saturated absorption line.

The laser excitation is pulsed. The blue laser power is $8 \mathrm{~mW}$. The red intensity is adjusted between $100 \mathrm{nW}$ and $2 \mu \mathrm{W}$, so that $\simeq 0.3$ Rydberg atom is produced by each pulse. We thus avoid dipole-dipole effects, which are not the focus of this study. The largest two-photon Rabi frequency is $27 \mathrm{kHz}$. Up to 300 excitation pulses, at a $3 \mathrm{~ms}$ time interval, are sent on the same atomic cloud without notable degradation of the trap. The atomic cloud is renewed every 8 seconds.

The Rydberg atoms are detected by field-ionization [Fig. 1(c)]. A voltage ramp $V_{i}$ is applied on the electrode $I$ facing the chip (kept at the ground voltage). The resulting field reaches at different times the ionization thresholds of the Rydberg levels $(37 \mathrm{~V} / \mathrm{cm}$ for $60 \mathrm{~S})$. The ions are accelerated and deflected (electrode $D$ ) towards a channeltron counter (Sjuts Optotechnik KBL 10 RSEDR). The detection efficiency is $90 \pm 10 \%$. It is calibrated by comparing the number of detected Rydberg atoms to the corresponding decrease in the number of trapped atoms. Before the ionization ramp, we minimize the $E_{y}$ field component by a applying a compensation voltage $V_{c}$ on the ionization electrode $I$.

The first laser spectroscopy with the chip's gold front mirror confirmed the stray field problem [29, 33]. Figure 2 present a laser spectrum in a MOT at $y=550 \mu \mathrm{m}$ (black points). The linewidth is $30 \mathrm{MHz}$ in spite of the $E_{y}$ minimization (application of a $3.7 \mathrm{~V} / \mathrm{cm}$ compensation field). It increases up to $40 \mathrm{MHz}$ in a 40 minutes time interval, while the line center drifts by $12 \mathrm{MHz}$ (red/gray points). Note that the Zeeman effect due to the MOT quadrupolar field is much smaller than the observed linewidth. These 
measurements point to inhomogeneous fields created by a $\mathrm{Rb}$ deposit onto the gold mirror. Using the cloud size $(\approx 200 \mu \mathrm{m})$ and the $-90 \mathrm{MHz} /(\mathrm{V} / \mathrm{cm})^{2}$ quadratic Stark shift of the $60 \mathrm{~S}$ level, we estimate the field gradient to be $\approx 12 \mathrm{~V} / \mathrm{cm}^{2}$. This value is incompatible with the use of atom chips for the dipole blockade, not to mention quantum information manipulations.

Stray fields may be reduced if the cold Rubidium atoms adsorb onto a $\mathrm{Rb}$ metallic mirror, which is an excellent optical reflector at $780 \mathrm{~nm}$ [36]. We have installed two $\mathrm{Rb}$ dispensers (SAES Getters 5G0125), thermally isolated from the chip and aiming directly at its surface (shields protect other parts, including the channeltron and the optical windows). After cooldown at $4.2 \mathrm{~K}$, the dispensers were activated for a few minutes. In spite of their high operating temperature, they did not heat the experiment above $12 \mathrm{~K}$. Rb atoms thus rapidly stick onto the chip surface. Using the dispensers' capacity and geometrical considerations, we expect that the Rb layer is nearly uniform over the chip, with a $\approx 90 \mathrm{~nm}$ thickness.

The blue triangles in Fig. 2 present an optical excitation spectrum recorded after this deposition and the optimization of $V_{c}$ in a magnetic trap at $y=670 \mu \mathrm{m}, z=0$. The width is reduced down to $1.7 \mathrm{MHz}$, mainly limited by laser linewidth. The center of the line is quite close to the expected position in zero field (dotted vertical line). This result evidences the dramatic improvement provided by the $\mathrm{Rb}$ coating on the optical spectrum. The line remained stable for months, provided the set-up is always kept at a low temperature, precluding any desorption.

We now use microwave spectroscopy in the smaller and colder magnetic trap to investigate the coherence of Rydberg level superpositions. We first estimate the residual electric field by studying the one-photon transitions $60 S_{1 / 2}, m_{J}=1 / 2 \rightarrow 60 P_{3 / 2}, m_{J}=3 / 2$ and $m_{J}=-1 / 2$ at $17.29 \mathrm{GHz}$. Their degeneracy is lifted by the $B_{x}$ trap magnetic field and they have a high Stark polarizability [about $\left.-500 \mathrm{MHz} /(\mathrm{V} / \mathrm{cm})^{2}\right]$. The microwave pulse is produced by a frequency-stabilized Anritsu generator and sent on the atoms through an optical access.

We recorded the microwave spectra for $y$ between 150 and $675 \mu \mathrm{m}$ and $z=0$. For each $y$ value, we minimize $E_{y}$ by adjusting $V_{c}$ to get the highest transition frequency. The resulting compensation field, found to be independent on $y$, is $0.09(2) \mathrm{V} / \mathrm{cm}$, nearly two orders of magnitude smaller than that observed before Rubidium deposition. Fig. 3(a) presents the spectrum of the two transitions at $y=455 \mu \mathrm{m}$. Their common zero field frequency is known within $50 \mathrm{kHz}$ from direct quantum defect measurements [37]. The microwave pulse duration is $20 \mu \mathrm{s}$ and the FWHM of the resonances $\approx 1.4 \mathrm{MHz}$.

From the position of the two lines and the known Zeeman and Stark effects, we deduce the $B_{x}$ magnetic field and the uncompensated $E_{\|}$electric field component parallel to the chip surface. The magnetic field $\left(B_{x}=8.71\right.$ Gauss for $y=455 \mu \mathrm{m}$ ) agrees within $\simeq 2 \%$ with the re-
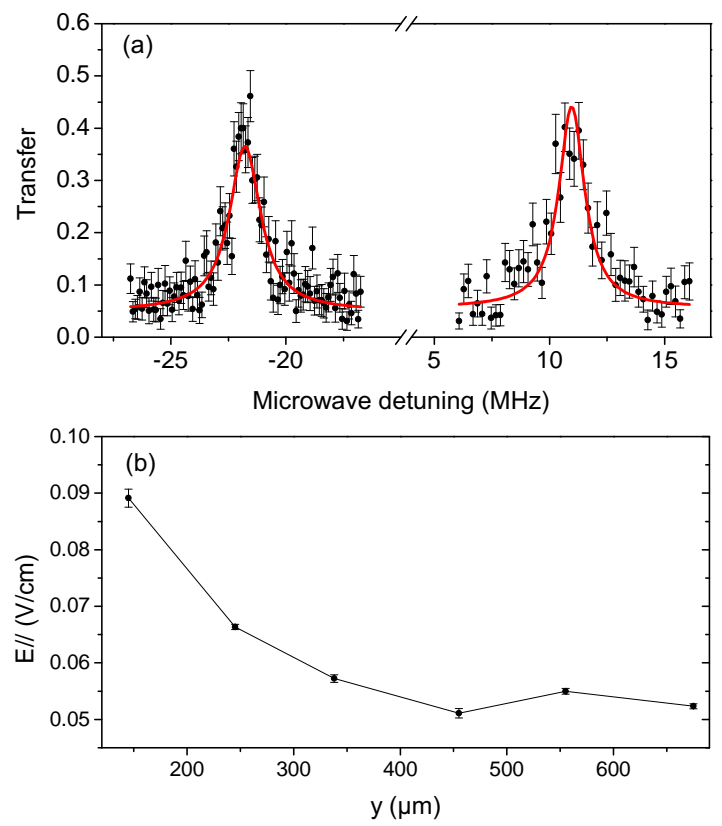

FIG. 3. (color online) (a) Spectroscopy of the $60 S_{1 / 2}, m_{J}=$ $1 / 2 \rightarrow 60 P_{3 / 2}, m_{J}=-1 / 2$ (left) and $m_{J}=3 / 2$ (right) transitions in the magnetic trap at $y=455 \mu \mathrm{m}$ from the chip. The transfer probability is shown as a function of the microwave detuning w.r.t the zero field line position. The points are experimental, with statistical standard deviation error bars. The red lines are Lorentzian fits. (b) Parallel electric field modulus, $\left|E_{\|}\right|$, with respect to the atom-chip distance $y$.

sult of RF spectroscopy. Figure 3(b) gives $\left|E_{\|}\right|$versus $y$. The uncompensated field component is largest close to the chip, and always below $0.1 \mathrm{~V} / \mathrm{cm}$.

We investigate the coherence time for a Rydberg atom qubit by focusing on the $60 S \rightarrow 61 S$ two-photon transition, at $2 \times 17.427 \mathrm{GHz}$, whose quadratic Stark shift is $-10.9 \mathrm{MHz} /(\mathrm{V} / \mathrm{cm})^{2}$. We operate at $y=455 \mu \mathrm{m}$ and adjust the $z$ position at $-350 \mu \mathrm{m}$, by minimizing the linewidth. Figure 4(a) presents the line obtained with a $300 \mu$ s microwave pulse. The width is $6.6 \mathrm{kHz}$, corresponding to a very encouraging coherence time. However, this line could be broadened by noise on $V_{c}$.

Ramsey spectroscopy [38], which is less sensitive to this noise, provide a better estimate of the transverse relaxation time, $T_{2}^{*}$. We apply to the atoms two $\pi / 2 \mathrm{mi}-$ crowave pulses (duration $0.3 \mu \mathrm{s}$ ) separated by a variable time interval $T$. Figure 4(b) presents the signal as a function of the delay $T$. The oscillation of the Ramsey fringes is at the chosen $70 \mathrm{kHz}$ detuning between atomic transition and microwave. A simple model of field-gradientinduced decoherence leads to a Gaussian envelope for the fringe contrast decay. Using this model as a fitting function [solid line in Fig. 4(b)], we get a 1/e relaxation time $T_{2}^{*}=163 \mu \mathrm{s}$. It would correspond to a field gradient of $0.2 \mathrm{~V} / \mathrm{cm}^{2}\left(0.4 \mathrm{~V} / \mathrm{cm}^{2}\right)$ in the $x(z)$ direction, estimated from a cloud extension of $60 \mu \mathrm{m}(30 \mu \mathrm{m})$. 

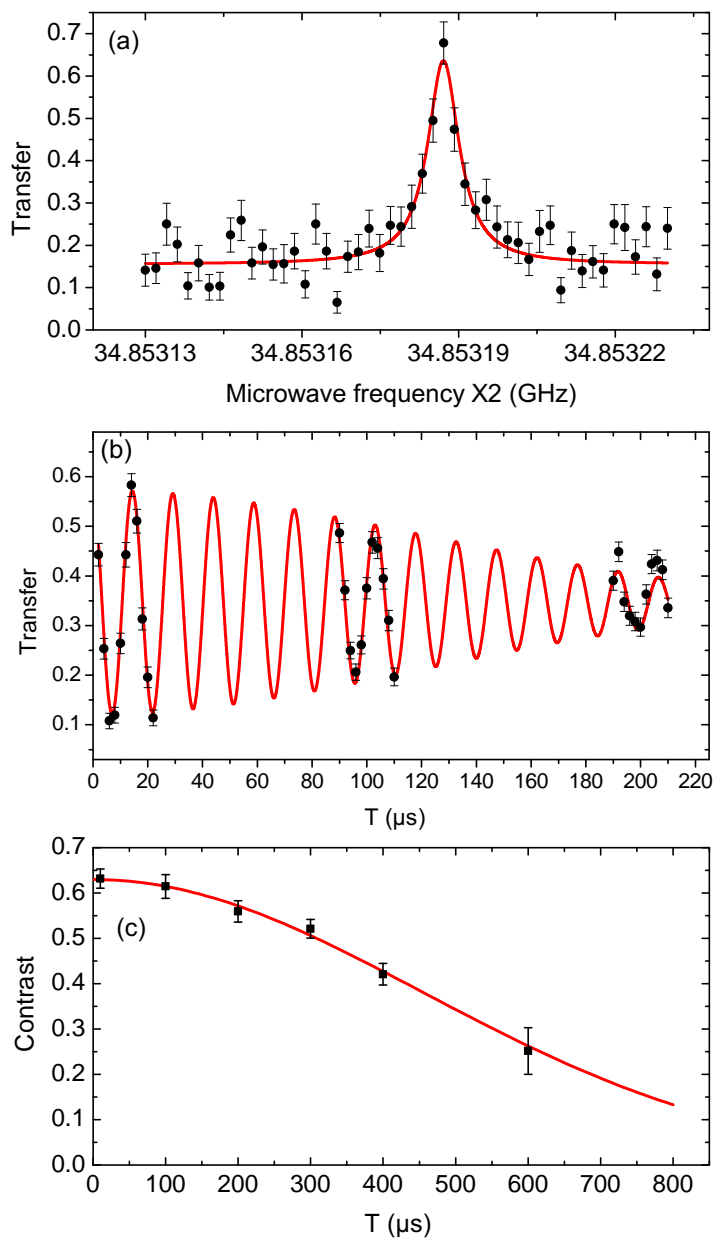

FIG. 4. (color online) (a) Spectroscopy of the $60 S \rightarrow 61 S$ two-photon transition. The transfer probability is shown as a function of twice the applied microwave frequency. The points are experimental with statistical standard deviation error bars. The line is a Lorentzian fit. (b) Ramsey spectroscopy of the $60 S \rightarrow 61 S$ transition at $y=455 \mu \mathrm{m}$ and $z=-350 \mu \mathrm{m}$. The transfer probability is shown as a function of the delay $T$ between the two $\pi / 2$ pulses. The dots are experimental, the red line is a fit. The error bars are the statistical standard deviation. (c) Contrast of the spin echo experiment as a function of the total duration $T$ of the sequence. The line is a Gaussian fit.

The longer coherence time is comparable to the lifetime of the $60 S$ Rydberg level, measured to be $210 \mu \mathrm{s}$ by monitoring the decay of the ionization signal. This lifetime is only slightly shorter than the theoretical value $(240 \mu \mathrm{s})$ at zero temperature. It is mainly determined by direct decay to the $5 P$ level by emission of a UV photon and by thermally induced microwave transitions to the closest $P$ Rydberg states. We infer from the measured lifetime an effective microwave temperature of $36 \mathrm{~K}$.

Stray field gradients reduce the Ramsey coherence time. We access the homogeneous transverse relaxation time, $T_{2}$, trough a spin echo sequence. At time $T / 2$, we apply a $\pi$ microwave pulse, resulting in a revival of the
Ramsey signal at time $T$. Figure 4(c) presents the contrast of this revival as a function of $T$ for $y=455 \mu \mathrm{m}$ and $z=-350 \mu \mathrm{m}$, together with a Gaussian fit. The coherence lifetime (at $1 / e$ contrast) is $T_{2}=631 \mu \mathrm{s}$, nearly thrice the atomic state lifetime (at $y=150 \mu \mathrm{m}$, we still observe $T_{2}=116 \mu \mathrm{s}$ ). Obviously, we detect, as in the Ramsey experiment, only those atoms that have survived over $T$. This explains why the statistical error bars get larger when $T$ increases. This coherence time is nearly two orders of magnitude larger than those observed on previous spectroscopic investigations of the microwave spectra in similar conditions [39]. The coherence is likely to be limited by the residual motion in the field gradients and by the noise on $V_{c}$ at a frequency of the order of $1 / T$. Taking into account the above estimations of the gradients, we infer that the electrical noise dominates.

In conclusion, we have observed near a superconducting atom chip Rydberg levels coherence times exceeding their lifetime. The stray electric field gradients due to adsorbed Rubidium atoms have been strongly reduced by covering the whole chip with a metallic Rubidium layer. This layer is quite stable in a cryogenic environment.

These results are extremely encouraging for the observation of the dipole blockade in this context. All experiments reported here have been performed with a low density of Rydberg atoms. We are now investigating density effects, which will be reported elsewhere.

This makes us confident that selected Rydberg atom numbers could be produced on-chip, opening the way to interesting quantum simulations of condensed matter problems $[4,5]$. On the longer terms, the route towards a hybrid quantum information processing architecture based on circuit QED and Rydberg atoms remains open [17-19].

This research has been supported by the EU Marie Curie Action CCQED, Project 264666, by the EU ICT Project SIQS Number 600645 and by the DECLIC ERC project.

[1] T. F. Gallagher, Rydberg Atoms (Cambridge University Press, Cambridge, 1994).

[2] S. Haroche and J.-M. Raimond, Exploring the quantum: atoms, cavities and photons (Oxford University Press, 2006).

[3] M. Saffman, T. G. Walker, and K. Mølmer, Rev. Mod. Phys. 82, 2313 (2010).

[4] M. Müller, S. Diehl, G. Pupillo, and P. Zoller, in Advances in Atomic, Molecular, and Optical Physics, volume 61, edited by E. A. Paul Berman and C. Lin (Academic Press, 2012), pp. $1-80$.

[5] H. Weimer, M. Muller, I. Lesanovsky, P. Zoller, and H. P. Buchler, Nat Phys 6, 382 (2010).

[6] R. Löw, H. Weimer, J. Nipper, J. B. Balewski, B. Butscher, H. P. Bächler, and T. Pfau, Journal of Physics B: Atomic, Molecular and Optical Physics 45, 
113001 (2012).

[7] M. D. Lukin, M. Fleischhauer, R. Côté, L. M. Duan, D. Jacksch, J. I. Cirac, and P. Zoller, Phys. Rev. Lett. 87, 037901 (2001).

[8] Y. O. Dudin and A. Kuzmich, Science 336, 887 (2012).

[9] M. Ebert, A. Gill, M. Gibbons, X. Zhang, M. Saffman, and T. G. Walker, Phys. Rev. Lett. 112, 043602 (2014).

[10] T. Pohl, E. Demler, and M. D. Lukin, Phys. Rev. Lett. 104, 043002 (2010).

[11] G. Pupillo, A. Micheli, M. Boninsegni, I. Lesanovsky, and P. Zoller, Phys. Rev. Lett. 104, 223002 (2010).

[12] P. Schausz, M. Cheneau, M. Endres, T. Fukuhara, S. Hild, A. Omran, T. Pohl, C. Gross, S. Kuhr, and I. Bloch, Nature 491, 87 (2012).

[13] T. Wilk, A. Gaëtan, C. Evellin, J. Wolters, Y. Miroshnychenko, P. Grangier, and A. Browaeys, Phys. Rev. Lett. 104, 010502 (2010).

[14] L. Isenhower, E. Urban, X. L. Zhang, A. T. Gill, T. Henage, T. A. Johnson, T. G. Walker, and M. Saffman, Phys. Rev. Lett. 104, 010503 (2010).

[15] O. Firstenberg, T. Peyronel, Q.-Y. Liang, A. V. Gorshkov, M. D. Lukin, and V. Vuletic, Nature 502, 71 (2013).

[16] M. H. Devoret and R. J. Schoelkopf, Science 339, 1169 (2013).

[17] D. Petrosyan, G. Bensky, G. Kurizki, I. Mazets, J. Majer, and J. Schmiedmayer, Phys. Rev. A 79, 040304 (2009).

[18] S. D. Hogan, J. A. Agner, F. Merkt, T. Thiele, S. Filipp, and A. Wallraff, Phys. Rev. Lett. 108, 063004 (2012).

[19] J. D. Pritchard, J. A. Isaacs, M. A. Beck, R. McDermott, and M. Saffman, Phys. Rev. A 89, 010301 (2014).

[20] A. Emmert, A. Lupascu, G. Nogues, M. Brune, J.-M. Raimond, and S. Haroche, Eur. Phys. J. D 51, 173 (2009).

[21] G. Nogues, C. Roux, T. Nirrengarten, A. Lupascu, A. Emmert, M. Brune, J.-M. Raimond, S. Haroche, B. Placais, and J. J. Greffet, EPL 87, 13002 (2009).

[22] C. Roux, A. Emmert, A. Lupascu, T. Nirrengarten,
G. Nogues, M. Brune, J.-M. Raimond, and S. Haroche, EPL 81, 56004 (2008).

[23] S. E. Anderson, K. C. Younge, and G. Raithel, Phys. Rev. Lett. 107, 263001 (2011).

[24] P. Hyafil, J. Mozley, A. Perrin, J. Tailleur, G. Nogues, M. Brune, J.-M. Raimond, and S. Haroche, Phys. Rev. Lett. 93, 103001 (2004).

[25] C. Fabre, P. Goy, and S. Haroche, J. Phys. B10, L183 (1977).

[26] C. Kocher and C. Taylor, Phys. Lett. A 124, 68 (1987).

$[27]$ T. Thiele, S. Filipp, J. A. Agner, H. Schmutz, J. Deiglmayr, M. Stammeier, P. Allmendinger, F. Merkt, and A. Wallraff, Phys. Rev. A 90, 013414 (2014).

[28] J. M. McGuirk, D. M. Harber, J. M. Obrecht, and E. A. Cornell, Phys. Rev. A 69, 062905 (2004).

[29] K. S. Chan, M. Siercke, C. Hufnagel, and R. Dumke, Phys. Rev. Lett. 112, 026101 (2014).

[30] A. Tauschinsky, R. M. T. Thijssen, S. Whitlock, H. B. van Linden van den Heuvell, and R. J. C. Spreeuw, Phys. Rev. A 81, 063411 (2010).

[31] R. P. Abel, C. Carr, U. Krohn, and C. S. Adams, Phys. Rev. A 84, 023408 (2011).

[32] J. D. Carter and J. D. D. Martin, Phys. Rev. A 83, 032902 (2011).

[33] H. Hattermann, M. Mack, F. Karlewski, F. Jessen, D. Cano, and J. Fortágh, Phys. Rev. A 86, 022511 (2012).

[34] L. A. Jones, J. D. Carter, and J. D. D. Martin, Phys. Rev. A 87, 023423 (2013).

[35] J. B. Balewski, A. T. Krupp, A. Gaj, D. Peter, H. P. Buchler, R. Low, S. Hofferberth, and T. Pfau, Nature 502, 664 (2013).

[36] N. V. Smith, Phys. Rev. B 2, 2840 (1970).

[37] W. Li, I. Mourachko, M. W. Noel, and T. F. Gallagher, Phys. Rev. A 67, 052502 (2003).

[38] N. F. Ramsey, Molecular Beams, International Series of Monographs on Physics (Oxford University Press, Oxford, 1985).

[39] J. D. Carter and J. D. D. Martin, Phys. Rev. A 88, 043429 (2013). 